Article

\title{
Design of Ku- and Ka-Band Flat Dual Circular Polarized Reflectarrays by Combining Variable Rotation Technique and Element Size Variation
}

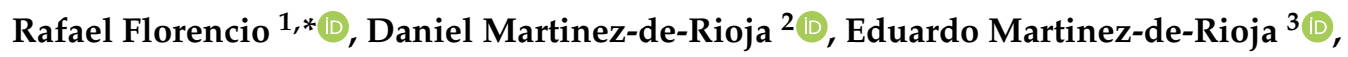 \\ Jose A. Encinar ${ }^{2}{ }^{(}$, Rafael R. Boix ${ }^{4}$ and Vicente Losada ${ }^{5}$ \\ 1 Department of Physics and Mathematics, University of Alcalá, 28805 Alcalá de Henares, Spain \\ 2 Information Processing and Telecommunications Center, Universidad Politécnica de Madrid (UPM), \\ 28040 Madrid, Spain; jd.martinezderioja@upm.es (D.M.-d.-R.); jose.encinar@upm.es (J.A.E.) \\ 3 Department of Signal Theory and Communications and Telematic Systems and Computing, \\ Universidad Rey Juan Carlos, 28943 Fuenlabrada (Madrid), Spain; eduardo.martinez@urjc.es \\ 4 Microwaves Group, Department of Electronics and Electromagnetism, College of Physics, \\ University of Seville, Avda. Reina Mercedes s/n, 41012 Seville, Spain; boix@us.es \\ 5 Microwaves Group, Department of Applied Physics 1, School of Computer Science Engineering, \\ University of Seville, Avda. Reina Mercedes s/n, 41012 Seville, Spain; losada@us.es \\ * Correspondence: rafaelflorenciodiaz@yahoo.es or rafael.florencio@uah.es
}

Received: 14 May 2020; Accepted: 10 June 2020; Published: 12 June 2020

check for updates

\begin{abstract}
In this work circularly polarized flat reflectarray antennas are designed to work at downlink frequencies in $\mathrm{Ku}$ - and Ka-band. The design approach for circular polarization radiation combines both the variable rotation technique (VRT) and the element size variation technique (ESVT). The reflectarray element employed consists of a rotable split ring operating at $12 \mathrm{GHz}$ (Ku-band) that surrounds two orthogonal sets of parallel dipoles of variable size operating at $20 \mathrm{GHz}$ (Ka-band). Rotations of split rings and different sizes of dipoles are exploited as degrees of freedom by VRT and ESVT for designing focused beams dual band dual circular polarized reflectarrays. The split ring can be used to achieve single focused beams for circular polarization by VRT at $12 \mathrm{GHz}$, and the set of orthogonal dipoles can be used to achieve two focused beams for dual circular polarization by ESVT at $20 \mathrm{GHz}$. In this way, the reflectarray element has been used to design two flat dual band circularly polarized reflectarrays that generate three pencil beams either in the same direction or in different directions: one pencil beam with right-hand circular polarization (RHCP) at $12 \mathrm{GHz}$ and two pencil beams with both RHCP and left-hand circular polarization (LHCP) at $20 \mathrm{GHz}$. The designed reflectarrays are carried out under local periodicity assumption by means of in-house electromagnetic software that applies the Method of Moments in the Spectral Domain. Validations of the in-house electromagnetic software show significant CPU time savings with respect to CPU time consumption provided by commercial software of general purpose as CST electromagnetics software. Numerical results of radiation patterns produced by the designed reflectarrays show efficiencies around $65 \%$ and a bandwidth of $6 \%$ for a main beam cross-polarization discrimination of $25 \mathrm{~dB}$. The numerical results are satisfactorily validated by CST software and a tolerance errors study has been numerically carried out with acceptable results.
\end{abstract}

Keywords: circular polarization; coupled dipoles; split-loops; reflectarrays; dual-band; dual-polarized antennas 


\section{Introduction}

For satcom-on-the-move (SOTM) applications, there is an increasing interest in developing Kuand Ka-band antennas that share a single aperture. Conventional solutions use bulky parabolic reflector antennas with very complex feeding systems [1-3]. Reflectarrays are an interesting alternative to reflector antennas owing to their low profile, light weight, radiation performance versatility, ease of manufacture, etc. [4]. Single aperture reflectarray antennas have been designed that are capable to provide coverage in $\mathrm{Ku}$ - and Ka-band with polarization agility [5-7]. In particular, a two-layered reflectarray antenna was presented in [7], which provides dual linear polarization in Ku- and Ka-band for a satellite downlink. Two stacked reflectarrays separated by a double screen frequency selective surface are used in [8] to implement an antenna that provides dual linear polarization at Ku-band for transmit (TX) and receive (RX), and single circular polarization at Ka-band for TX and RX. The impressive performance of this latter antenna is achieved at the expense of a complex multilayered structure with four metallization levels [8].

In this work we focus on the design of a circular polarization reflectarray antenna operating at $\mathrm{Ku}$ - and Ka-bands for a satellite downlink. By comparison with the antennas presented in $[7,8]$ that mainly operate in linear polarization, circular polarization is chosen since it is more robust to antenna orientation changes. Two different techniques are combined to achieve the correct phasing in circular polarization in each frequency band. The variable rotation technique (VRT), introduced by Huang and Pogorzelski [9], is employed at Ku-band to achieve singular circular polarization. This technique has been used in the past for the design of single band [10], and dual band circular polarization reflectarray antennas at Ka-band [11,12]. In [13] a circularly polarized tri-band and three pencil beam reflectarray is shown. This reflectarray operates at 1.6, 20.0 and $29.8 \mathrm{GHz}$ with right-hand circular polarization (RHCP), left-hand circular polarization (LHCP) and RHCP respectively. However, although the results are satisfactory, the proposed antenna is bulky and difficult to manufacture. The element size variation technique (ESVT) [14,15], is the phasing technique employed at Ka-band, and this technique makes it possible to operate the antenna in dual circular polarization. A dual circular polarization reflectarray based on the ESVT was designed in Section III.B of [16], and the VRT was subsequently used to separate the beams of the orthogonal circular polarizations (see section III.C of [16]). However, the designed antenna only works at single frequency band. The reflectarray element employed in our work consists of a split ring surrounding two orthogonal sets of dipoles on a two-layered substrate. The split ring is used to achieve single focused beam for circular polarization by VRT at $12 \mathrm{GHz}$ as in [11], and the set of orthogonal dipoles is used to achieve two focused beams for dual circular polarization by ESVT at $20 \mathrm{GHz}$ as in section III.B of [16]. The reflectarray element used in this paper was already introduced in [17] as a potential element to achieve dual band dual circular polarization at Ka-band for TX and RX. However, reflectarray antenna designs were not provided in [17]. In [18] this element was used to show the feasibility of a reflectarray design for the separation of two unfocused beams in dual-band for orthogonal circular polarizations by means of VRT using a single feeder. Although successful experimental results were shown in [18] to validate the proof of the concept, VRT was only used for the separation of two unfocused beams. Therefore, variation size and rotations capabilities of the reflectarray element were not exploited as degrees of freedom to focus the reflected beams by means of ESVT and VRT. The mean novelty of this work shows that both the rotations of split rings and the different sizes of dipoles are exploited as degrees of freedom by VRT and ESVT for designing focused beams dual band dual circular polarized reflectarrays for $\mathrm{Ku}$ - and Ka-band satellite downlink applications. We provide two different antenna designs that show the feasibility of the element in Ku- and Ka-band satellite downlink applications for circular polarization. Each designed antenna provides single circular polarization radiation at $12 \mathrm{GHz}$ by variable rotation capability of the element, and dual circular polarization radiation at $20 \mathrm{GHz}$ by variable size capability of the element, either in the same beam direction at both frequencies or in different beam directions. Since successful experimental validations was shown in $[17,18]$ using the same reflectarray element for different applications, numerical results will be only shown in this work. Promising results for $\mathrm{Ku}-$ 
and Ka-band satellite downlink applications for circular polarization are obtained. Such promising results suggest that proposed architecture could be applied for 5G and beyond applications [19].

\section{Reflectarray Element}

The reflectarray element used in this work is based on a two-layered substrate which hosts two levels of metallization, as shown in Figure 1a,b.

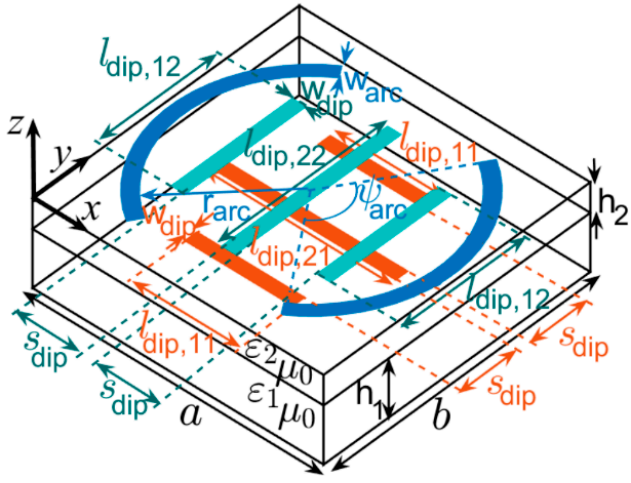

(a)

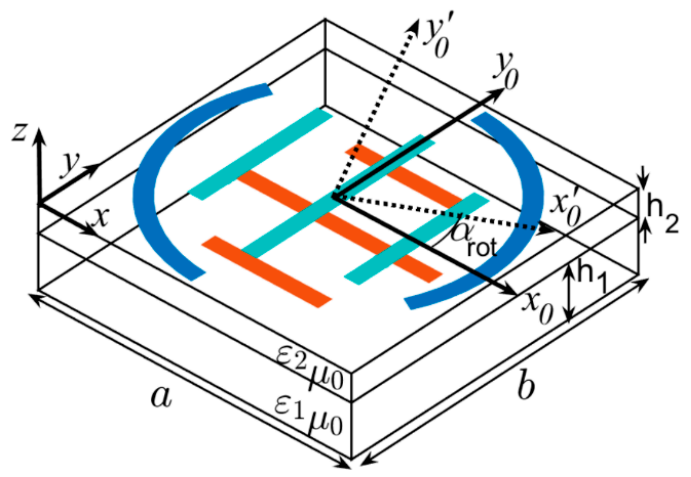

(b)

Figure 1. (a) Reflectarray element based on two orthogonal sets of parallel dipoles surrounded by a non-rotated split ring. (b) Reflectarray element with rotated split ring and definition of rotation angle $\alpha_{\text {rot }}$.

The element consists of a split ring that surrounds two orthogonal sets of parallel dipoles. The split ring is printed on the upper side of the upper dielectric layer, and the sets of dipoles are printed on both opposite sides of this dielectric layer. The split ring is aimed at the design of the antenna at $12 \mathrm{GHz}$, and the parallel dipoles are used for the design at $20 \mathrm{GHz}$. A bottom dielectric layer is introduced as a separator between the upper layer and the ground plane. The material used for the upper layer and the separator is a commercial material with low RF losses, Rogers Duroid 5880, which means that the multilayered substrate supporting the reflectarray element is composed of two dielectric layers with thickness $h_{\mathrm{i}}$ and complex permittivity $\varepsilon_{i}=\varepsilon_{0} \varepsilon_{r i}\left(1-j \tan \delta_{i}\right)-i=1$,2- (see Figure 1 ), where $\varepsilon_{r 1}=\varepsilon_{r 2}=2.2$, $\tan \delta_{1}=\tan \delta_{2}=0.0009, h_{1}=1.524 \mathrm{~mm}, h_{2}=0.787 \mathrm{~mm}$ and $\varepsilon_{0}$ the vacuum permittivity. The relative permittivity and loss tangent values are provided by [20] at $10 \mathrm{GHz}$. A period $a=b=8 \mathrm{~mm}$ (which is roughly $0.533 \lambda$ at $20 \mathrm{GHz}$ ) has been selected for the unit cell of the reflectarray to provide both enough room for the dipoles and the split ring and, at the same time, to avoid the appearance of grating lobes. The center of central dipoles on each side of the upper dielectric layer are located at the center of each side of the dielectric layer of cell and the lateral dipoles are symmetrically separated from the central dipole (see Figure 1a). In similar way, the center of the circular arcs are located at the center of the cell and these arcs are symmetrical with respect to the mirror symmetry axis $y^{\prime} 0$ (see Figure 1b). The geometrical parameters of layout have been adjusted to provide a smooth and linear behavior of the phase-shift for variations of both the arc lengths of the split ring at $12 \mathrm{GHz}$, and the dipoles lengths at $20 \mathrm{GHz}$. The fixed final dimensions chosen are relation of lengths between the dipoles $l_{d i p, 11}=0.6 l_{d i p, 21}, l_{d i p, 12}=0.73 l_{d i p, 22}$, the widths of dipoles and arcs $\mathrm{w}_{\text {dip }}=\mathrm{w}_{\text {arc }}=0.2 \mathrm{~mm}$, separation between dipoles $s_{\text {dip }}=1.8 \mathrm{~mm}$, and radius of arcs $r_{\text {arc }}=3.575 \mathrm{~mm}$. The lengths $l_{d i p, 21}$ and $l_{d i p, 22}$ of central dipoles printed on each side of the upper dielectric layer will be the geometrical design parameters for reflectarray design process at $20 \mathrm{GHz}$. The rotation angle $\alpha_{\text {rot }}$ of the mirror symmetry axis of the split-ring (see Figure $1 \mathrm{~b}$ ) and the subtended angle $\psi_{\text {arc }}$ by extremes of the arcs of the split rings (see Figure 1a) will be the geometrical design parameters for reflectarray design process at $12 \mathrm{GHz}$. 
An in-house electromagnetic software that applies the Method of Moments in the Spectral Domain (MoM-SD) is used for the analysis of the reflectarray cell with rotated split ring and two orthogonal sets of parallel dipoles of Figure $1 \mathrm{~b}$ in a periodic environment [21,22] (i.e., periodic boundary conditions are considered) illuminated with linearly or circular polarized plane wave (i.e., TEz, TMz polarization or combination). The incidence direction of the plane wave is given by the spherical angular coordinates $\theta_{i}$ and $\phi_{i}$. According with [21,22], in the method of moment in spectral domain approach [23], the multilayer medium is modelled by means of bidimensional spectral multilayer dyadic Green functions [24] which takes into account permittivity, losses tangent and thickness of each dielectric layer of the multilayer medium and the ground plane as perfect electric conductors of negligible thickness. The layout is also modelled as perfect electric conductors of negligible thickness. Entire domain basis functions accounting for edge singularities are used in the approximation of the surface current density on split ring and dipoles as shown in [21,22] respectively. According with the results shown in [21,22], a house code based on MoM-SD with proposed edge singularities entire domain basis functions which lead CPU time consumptions of electromagnetics analysis of the element which are roughly 30-times faster than those obtained using CST frequency domain solver. Since a reflectarray has thousands of elements, the analysis tool of reflectarray element should be called tens of thousands of times in a reflectarray design. In this way, the house-code is more suitable than CST software for reflectarray designs.

The MoM-SD makes it possible to characterize the reflectarray cell of Figure $1 \mathrm{~b}$ by means of a reflection matrix $\mathbf{R}_{0}$ relating the complex $x_{0}$ and $y_{0}$ components (the $x_{0}$ and $y_{0}$ axes of Figure $1 \mathrm{~b}$ are parallel to the dipoles) of the reflected electric field and the incident electric field for a plane wave impinging on the cell in a direction given by the angular spherical coordinates $\theta_{\mathrm{i}}$ and $\phi_{\mathrm{i}}$. The components of $\mathbf{R}_{0}$ are defined in Equation [16]:

$$
\left(\begin{array}{c}
E_{x}^{r e f} \\
E_{y}^{r e f}
\end{array}\right)=\left(\begin{array}{ll}
R_{0, x x} & R_{0, x y} \\
R_{0, y x} & R_{0, y y}
\end{array}\right) \cdot\left(\begin{array}{c}
E_{x}^{i n c} \\
E_{y}^{i n c}
\end{array}\right)
$$

The components of the matrix $\mathbf{R}_{0}$ are complex numbers. The phase of the complex numbers of diagonal components of matrix $\mathbf{R}_{0}$ are key in reflectarray designs. In the same way, a matrix $\mathbf{R}_{0^{\prime}}$ can be defined relating the complex $x_{0}^{\prime}$ and $y_{0}^{\prime}$ components of the reflected and incident field, where the axes $x_{0}^{\prime}$ and $y_{0}^{\prime}$ of Figure $1 \mathrm{~b}$ are obtained by rotating the axes $x_{0}$ and $y_{0}$ an angle $\alpha_{\text {rot }}$ around the $z$ axis $\left(\alpha_{\text {rot }}\right.$ is the rotation angle of the split ring between Figure $1 \mathrm{a}, \mathrm{b})$. $\mathbf{R}_{0^{\prime}}$ can be obtained in terms of $\mathbf{R}_{0}$ as shown below [16]:

$$
\left(\begin{array}{ll}
R_{0, x x}^{\prime} & R_{0, x y}^{\prime} \\
R_{0, y x}^{\prime} & R_{0, y y}^{\prime}
\end{array}\right)=\left(\begin{array}{cc}
\cos \left(\alpha_{r o t}\right) & \sin \left(\alpha_{r o t}\right) \\
-\sin \left(\alpha_{r o t}\right) & \cos \left(\alpha_{r o t}\right)
\end{array}\right) \cdot\left(\begin{array}{ll}
R_{0, x x} & R_{0, x y} \\
R_{0, y x} & R_{0, y y}
\end{array}\right)\left(\begin{array}{cc}
\cos \left(\alpha_{r o t}\right) & -\sin \left(\alpha_{r o t}\right) \\
\sin \left(\alpha_{r o t}\right) & \cos \left(\alpha_{r o t}\right)
\end{array}\right)
$$

Since the components of matrix $\mathbf{R}_{0}$ are complex numbers, the components of matrix $\mathbf{R}_{0^{\prime}}$ are also complex numbers. A third matrix $\mathbf{R}^{\mathrm{c}}$ can be defined which relates the LHCP and RHCP complex components of the reflected and incident field for the impinging wave as shown below [16]:

$$
\left(\begin{array}{c}
E_{R H C P}^{r e f} \\
E_{L H C P}^{r e f}
\end{array}\right)=\left(\begin{array}{ll}
R_{R H C P, R H C P} & R_{R H C P, L H C P} \\
R_{L H C P, R H C P} & R_{L H C P, L H C P}
\end{array}\right) \cdot\left(\begin{array}{l}
E_{R H C P}^{i n c} \\
E_{L H C P}^{i n c}
\end{array}\right)
$$

It turns out that $\mathbf{R}^{\mathbf{c}}$ can be also easily obtained in terms of $\mathbf{R}_{\mathbf{0}}$ by means of the transformations shown in [25]. Since the components of matrix $\mathbf{R}_{0}$ are complex numbers, the components of matrix $\mathbf{R}^{\mathbf{c}}$ are also complex number.

Huang and Pogorzelski demonstrated that the phases of the coefficients $R_{R H C P, R H C P}$ and $R_{L H C P, L H C P}$ defined in (3) have a linear dependence on the rotation angle $\alpha_{\text {rot }}$ defined in Figure $1 \mathrm{~b}$ provided 
$R_{0, x x}^{\prime}=-R_{0, y y}^{\prime}$ (i.e., the gap between the phases of the complex numbers $R_{0, x x}^{\prime}$ and $R_{0, y y}^{\prime}$ is $180^{\circ}$ ). This linear dependence can be written as:

$$
\angle R_{R H C P, R H C P}=\angle R_{0, x x}^{\prime}+2 \alpha_{\text {rot }} ; \angle R_{L H C P, L H C P}=\angle R_{0, x x}^{\prime}-2 \alpha_{r o t}
$$

Moreover, under this condition, the cross-polar coefficients $R_{R H C P, L H C P}$ and $R_{L H C P, R H C P}$ are negligible. Equation (4) shows the dependence with respect to the rotation angle $\alpha_{\text {rot }}$ of phase-shift produced by the reflectarray cell in the phase of the circularly polarized electric field of the reflected wave. According with Equation (4) this dependence is linear with respect to the rotation angle $\alpha_{\text {rot }}$. Moreover, if the rotation angle $\alpha_{\text {rot }}$ is varied from $-90^{\circ}$ to $+90^{\circ}$, the phase-shift range is a cycle of $360 \mathrm{deg}$. So, any required phase shifts for the elements of a circularly polarized reflectarray antenna can be adjusted by simply rotating the elements of the antenna an adequate amount [9]. This is called the VRT. In this way, the subtended angle of $\psi_{\text {arc }}$ will be adjust to preserve the condition $R_{0, x x}^{\prime}=-R_{0, y y}^{\prime}$ while that the rotation angle $\alpha_{\text {rot }}$ will be adjust to match the phase shifts $\angle R_{R H C P, R H C P}$ to the required phase shift for right-hand circular polarization in each reflectarray element at $12 \mathrm{GHz}$. On the other hand, the condition $R_{0, x x}=-R_{0, y y}$ is preserved at $20 \mathrm{GHz}$ by adjustment of the lengths $l_{d i p, 21}$ and $l_{d i p, 22}$ of central dipoles of each orthogonal set without rotations. Under this condition, Equation (4) is satisfied but with $\alpha_{r o t}=0$ since the dipoles are not rotated (i.e., equation $\angle R_{R H C P, R H C P}=\angle R_{R H C P, R H C P}=\angle R_{0, x x}$ is satisfied for the dipoles at $20 \mathrm{GHz}$ ). So, as the length of dipole with direction parallel to $x_{0}$ axis, $l_{\text {dip,21 }}$ is varied, the value of $\angle R_{0, x x}$ is changed. According with [26,27], 360 deg of phase shift range of $\angle R_{0, x x}$ can be achieved by variation of lengths of three parallel dipoles using an appropriated relation between lengths of lateral dipoles and the length of central dipole (in our case we have fixed the relations $\left.l_{d i p, 11}=0.6 l_{d i p, 21}, l_{d i p, 12}=0.73 l_{d i p, 22}\right)$. In this way, the lengths of dipoles lengths $l_{d i p, 21}$ and $l_{d i p, 22}$ will be adjusted for both, to preserve the condition $R_{0, x x}=-R_{0, y y}$ and to match the phase shifts $\angle R_{0, x x}$ to the required phase shift in each reflectarray element at $20 \mathrm{GHz}$.

Figure $2 \mathrm{~b}$ shows how $\psi_{\text {arc }}$ must be modified as $\alpha_{r o t}$ is varied to keep $R_{0, x x}^{\prime}=-R_{0, y y}^{\prime}$. And Figure 2c shows how the phases of $R_{R H C P, R H C P}$ and $R_{L H C P, L H C P}$ linearly vary with $\alpha_{r o t}$ in accordance with Equation (4) when the conditions of Figure $2 b$ are fulfilled. Our MoM-SD results of Figure $2 c$ have been compared with the results provided by the commercial software CST [28], and good agreement has been found between both sets of results. We would like to point out that the average CPU time consumption produced by in-house code is $0.77 \mathrm{~s}$ for each analysis of the phase curves shown in Figure 2c while that, the average CPU time consumption produced by CST frequency domain solver under unit cell boundary conditions and Floquet port is roughly $540 \mathrm{~s}$ for each analysis using adaptive mesh option. These CPU time consumptions are obtained in a laptop computer with processor Intel Core i7-6700HQ at $2.6 \mathrm{GHz}$ with $32 \mathrm{~GB}$ of RAM memory. 


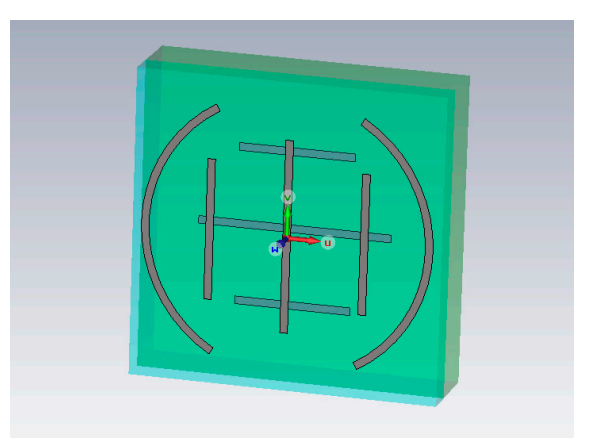

(a)



(b)



(c)

Figure 2. (a) 3D CST model picture. (b) Numerical adjusted values of $\psi_{\text {arc }}$ which preserve the condition $R_{0, x x}^{\prime}=-R_{0, y y}^{\prime}$ as a function of the rotation angle $\alpha_{r o t}$. (c) Simulated phase of the reflection coefficients $R_{R H C P, R H C P}$ and $R_{L H C P, L H C P}$ provided by the pairs $\left(\psi_{\text {arc }}, \alpha_{\text {rot }}\right)$ shown in (a) as a function of the rotation angle $\alpha_{\text {rot }}$. The numerical results are shown at $12 \mathrm{GHz}$ under incidence angles $\theta_{i}=19^{\circ}, \phi_{i}=0^{\circ}$ and fixed lengths of dipoles $l_{\mathrm{dip}, 21}=l_{\mathrm{dip}, 22}=5 \mathrm{~mm}$.

The results of Figure $2 b, c$ have been obtained for fixed lengths of the longest dipoles in Figure 1a,b, $l_{\text {dip }, 21}=l_{\text {dip,22 }}=5 \mathrm{~mm}$. However, these lengths have to be varied in order to make the element work for a circularly polarized reflectarray at $20 \mathrm{GHz}$, which will unavoidably affect the element performance at $12 \mathrm{GHz}$. Figure 3 a shows to which extent the condition $R_{0, x x}^{\prime}=-R_{0, y y}^{\prime}$ is lost when $l_{\text {dip,21 }}=l_{\text {dip,22 }}$ 
departs from $5 \mathrm{~mm}$, and Figure $3 \mathrm{~b}$ shows how this departure affects the magnitude of the non-diagonal cross-polar coefficients of the matrix $\mathbf{R}^{\mathrm{c}}$ in (3). Please note that the larger the departure of the phase difference from $-180^{\circ}$ in Figure 3a, the larger the cross-polarization in Figure 3b, which reaches a maximum of $-14 \mathrm{~dB}$ when $l_{d i p, 21}=l_{d i p, 22}=6 \mathrm{~mm}$ and $\alpha_{r o t}=0^{\circ}$.

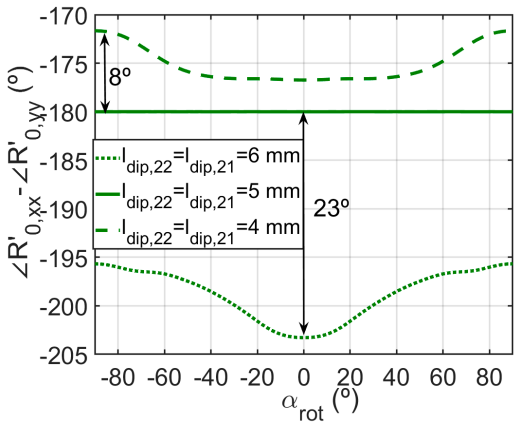

(a)



(b)

Figure 3. (a) Simulated difference between the phases of $R_{0, x x}^{\prime}$ and $R_{0, y y}^{\prime}$ provided by the pairs $\left(\psi_{\text {arc }}, \alpha_{\text {rot }}\right)$ shown in Figure 2a for different values of $l_{\text {dip,21 }}=l_{\text {dip,22. }}$ (b) Simulated magnitude of the cross-polar coefficient $R_{L H C P, R H C P}$ (provided by the pairs $\left(\psi_{\text {arc }}, \alpha_{\text {rot }}\right)$ shown in Figure 2a) as a function of the rotation angle $\alpha_{\text {rot }}$. The numerical results are shown at $12 \mathrm{GHz}$ under incidence angles $\theta_{i}=19^{\circ}$, $\phi_{i}=0^{\circ}$.

If we use the element of Figure 1a,b for the design of a circularly polarized antenna by ESVT at $20 \mathrm{GHz}$, the lengths of the dipoles $l_{d i p, 21}$ and $l_{d i p, 22}$ have to be adjusted to preserve the condition $R_{0, x x}=-R_{0, y y}$, which is necessary to keep the sense of circular polarization after reflection on the reflectarray antenna. Figure 4 a shows a color-map of the subtraction $\angle R_{0, x x}-\angle R_{0, y y}$ as a function of the dipole lengths $l_{d i p, 21}$ and $l_{d i p, 22}$. These results have been obtained for fixed values of $\psi_{\text {arc }}$ as $\alpha_{\text {rot }}$ (we have chosen $\psi_{\text {arc }}=118.9^{\circ}$ and $\alpha_{\text {rot }}=0^{\circ}$ in accordance with Figure 2a). Black solid lines in Figure 4a indicate the locus of pairs of dipole lengths that lead to the condition $R_{0, x x}=-R_{0, y y}$. Figure $4 \mathrm{~b}$ shows the phases of $R_{0, x x}$ and $R_{0, y y}$ obtained while using the pairs of dipole lengths defined by the black solid lines of Figure $4 \mathrm{a}$. These phase curves show a smooth behavior within a phase range of $388^{\circ}$ (larger than a cycle of $360^{\circ}$ ) where condition $R_{0, x x}=-R_{0, y y}$ is satisfied. The phase curves of $R_{R H C P, R H C P}$ and $R_{L H C P, L H C P}$ for circular polarization applications are also shown, which coincide with those of $R_{0, x x}$ since $\alpha_{\text {rot }}=0^{\circ}$ (see Equation (4) and Figure 1b). Since the phase curves for $R_{R H C P, R H C P}$ and $R_{L H C P, L H C P}$ coincide, this can be used to design a circular polarization reflectarray by means of ESVT that works for both RHCP and LHCP conditions as will be shown in the next Section. The phases curves obtained in Figure $4 \mathrm{~b}$ with our MoM-SD software have been compared with CST, and good agreement has been found. The average CPU time consumptions for analysis are similar to those obtained in the analysis shown in Figure 2. The CPU time consumptions are obtained in a laptop computer with processor Intel Core i7-6700HQ at $2.6 \mathrm{GHz}$ with $32 \mathrm{~GB}$ of RAM memory. 




(a)



(b)

Figure 4. (a) Color-map showing the simulated difference between the phases of $R_{0, x x}$ and $R_{0, y y}$ as a function of the dipole lengths $l_{\mathrm{dip}, 21}$ and $l_{\mathrm{dip}, 22}$. Black solid lines indicate the pairs of dipole lengths leading to the condition $R_{0, x x}=-R_{0, y y}$. (b) Simulated phases of $R_{0, x x}$ and $R_{0, y y}$ provided by the pairs of dipole lengths defined by the black solid lines of (a). The phases of $R_{R H C P, R H C P}$ and $R_{L H C P, L H C P}$ are also shown. These results are obtained at $20 \mathrm{GHz}$ under incidence angles $\theta_{i}=19^{\circ}, \phi_{i}=0^{\circ}$ when $\psi_{\text {arc }}=119^{\circ}$ and $\alpha_{\text {rot }}=0^{\circ}$.

As in the case shown in Figure 3, the conditions for circular polarization design at $20 \mathrm{GHz}$ shown in Figure 4 may change when $\psi_{\text {arc }}$ and $\alpha_{\text {rot }}$ are modified to adjust the phase shift of the element for circular polarization applications by VRT at $12 \mathrm{GHz}$. Figure 5a shows the difference between the phases of the $R_{0, x x}$ and $R_{0, y y}$ when the values of $\psi_{\text {arc }}$ and $\alpha_{\text {rot }}$ used in Figure 4 are varied. Additionally, Figure $5 \mathrm{~b}$ shows how this affects the cross-polar coefficient $R_{L H C P, R H C P}$. Please note that the increase in the magnitude of $R_{L H C P, R H C P}$ is much larger in Figure $5 \mathrm{~b}$ than in Figure $3 \mathrm{~b}$. In fact, $\left|R_{L H C P, R H C P}\right|$ reaches $-5 \mathrm{~dB}$ in Figure $5 \mathrm{~b}$ when $\psi_{\text {arc }}=109.8^{\circ}, \alpha_{\text {rot }}=45^{\circ}$ and $l_{d i p, 21}=5.9 \mathrm{~mm}$, which is due to the fact that the difference between the phases of $R_{0, x x}$ and $R_{0, y y}$ departs $78^{\circ}$ from $-180^{\circ}$ in Figure $5 \mathrm{a}$ (a maximum departure of $23^{\circ}$ is observed in Figure $3 a$ ). This means that the cross-polarization performance of a dual-band circular polarization reflectarray antenna based on the element of Figure 1 will be better if we first adjust the dimensions of the split ring by VRT at $12 \mathrm{GHz}$ and then fix the dipoles lengths by ESVT at $20 \mathrm{GHz}$, instead of first fixing the dipoles lengths at $20 \mathrm{GHz}$, and then adjusting the split rings at $12 \mathrm{GHz}$.



(a)



(b)

Figure 5. (a) Simulated difference between the phases of $R_{0, x x}$ and $R_{0, y y}$ for different values of $\psi_{\text {arc }}$ and $\alpha_{\text {rot }}$ (b) Simulated magnitude of the cross-polar coefficients $R_{L H C P, R H C P}$ for different values of $\psi_{\text {arc }}$ and $\alpha_{\text {rot }}$. The results are obtained for the pairs of lengths of dipoles $l_{\mathrm{dip}, 21}$ and $l_{\mathrm{dip}, 22}$ indicated by the black solid lines of Figure $4 \mathrm{a}$ at $20 \mathrm{GHz}$ under incidence angles $\theta_{i}=19^{\circ}, \phi_{i}=0^{\circ}$. 


\section{Reflectarray Design and Results}

A circular flat reflectarray which consists of 489 elements arranged in a $25 \times 25$ square grid with cell size of $8 \times 8 \mathrm{~mm}$ has been designed (the diameter of the antenna is $20 \mathrm{~cm}$ ). The elements are of the type shown in Figure 1b. The reflectarray is intended to radiate a RHCP focused beam in the direction $\phi_{\text {beam }}=0^{\circ}$ and $\theta_{\text {beam }}=19^{\circ}$ (see Figure 6) at $12 \mathrm{GHz}$, and two RHCP and LHCP focused beams in the same direction at $20 \mathrm{GHz}$. It is illuminated by a feed horn whose radiated pattern is modeled as $\cos ^{\mathrm{q}}(\theta)$ model for circular polarization [29]. In particular, the models are $\cos ^{10}(\theta)$ at $12 \mathrm{GHz}$ and $\cos ^{13}(\theta)$ at $20 \mathrm{GHz}$ (see [7] for details). The feed illuminates the edges of the reflectarray with a taper of $-12 \mathrm{~dB}$ at both frequencies, 12 and $20 \mathrm{GHz}$. Its face center is located at the coordinates $\left(x_{f}, y_{f}, z_{f}\right)=(-0.0639$, $0.0,0.185)(\mathrm{m})$ with respect to the coordinate system with origin at the center of the reflectarray that is shown in Figure 6.

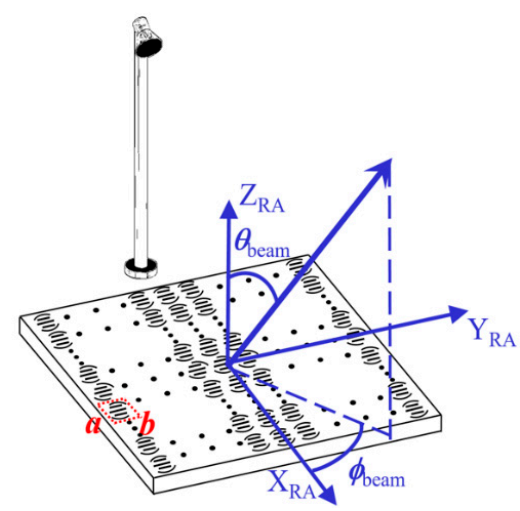

Figure 6. Reflectarray antenna picture. Coordinate system with origin at the reflectarray center and beam direction.

The reflectarray design is carried out in two stages. In the first stage we make use of Equation (4) to obtain the desired phase shift in each element according to Equation (3.3) of [4] at $12 \mathrm{GHz}$. The rotation angle $\alpha_{\text {rot }}$ and the arc angle $\psi_{\text {arc }}$ are adjusted so that $R_{0, x x}^{\prime}=-R_{0, y y}^{\prime}$ and (4) are fulfilled while $l_{d i p, 21}$ $=l_{d i p, 22}=5 \mathrm{~mm}$. Once the split ring parameters $\alpha_{\text {rot }}$ and $\psi_{\text {arc }}$ have been fixed for each reflectarray element, in the second stage the required phase shifts are determined at $20 \mathrm{GHz}$ by means of Equation (3.3) of [4]. For each pair values of $\alpha_{\text {rot }}$ and $\psi_{\text {arc }}$, curves similar to those of Figure 4 are generated in each element, and the dipoles lengths $l_{d i p, 21}$ and $l_{d i p, 22}$ are adjusted so that condition $R_{0, x x}=-R_{0, y y}$ is fulfilled. As commented in Section 2, the procedure based on first adjusting the split rings at $12 \mathrm{GHz}$, and subsequently adjusting the dipole lengths at $20 \mathrm{GHz}$, leads to lower cross-polarization values. Figure $7 \mathrm{a}, \mathrm{b}$ show the layouts printed on bottom and upper sides of the upper dielectric layer respectively. 




(a)

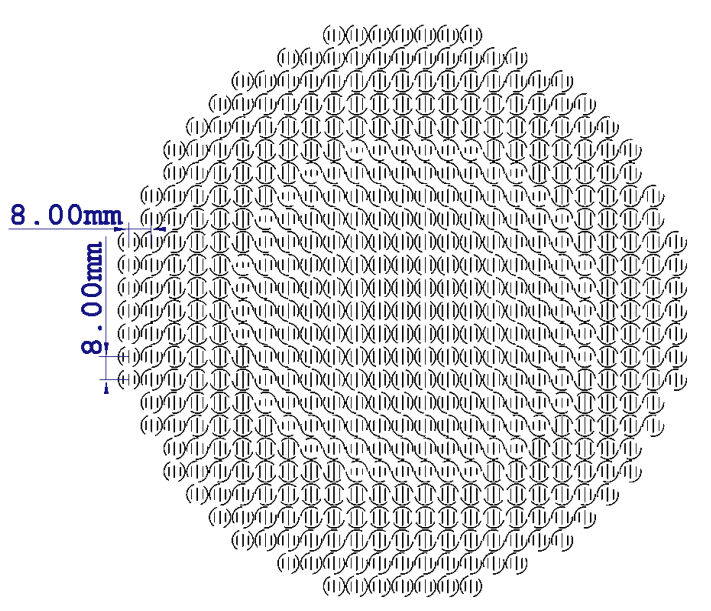

(b)

Figure 7. (a) Reflectarray layout printed on the bottom side of the upper dielectric layer. (b) Reflectarray layout printed on the upper side of the upper dielectric layer.

The designed reflectarray has been analyzed with both our MoM-SD code and CST under the local periodicity assumption (LPA) [4]. The radiation patterns have been computed in terms of the electric and magnetic currents on the reflectarray surface as shown in [30]. The numerical results obtained for cuts of the radiation patterns with elevation plane ( $y=0$ plane in coordinate system defined in Figure 6) and azimuth plane (constant plane which includes the direction of maximum of radiation and the $Y_{R A}$-axis defined in Figure 6) are shown in Figure 8a,b. Good agreement is found between our MoM-SD results and those provided by CST for the copolar components of the radiated field.

The overall performance of the antenna is summarized in Table 1, where cross-polar discrimination (XPD) is defined as the difference in $\mathrm{dB}$ between the copolar level and the cross-polar level at the pointing direction of the main beam. Note the cross-polar levels at $20 \mathrm{GHz}$ are much smaller than those at $12 \mathrm{GHz}$. This is logical since the design at $12 \mathrm{GHz}$ (first stage) did not use the exact final values of the dipole's lengths, but the design at $20 \mathrm{GHz}$ (second stage) used the exact final values of both the arc lengths/rotations and the dipole lengths. Figure $9 \mathrm{a}, \mathrm{b}$ show the maximum gain for co-polar and cross-polar radiated components versus frequency in each frequency band. We can check that the gain remains stable (variations less than $1 \mathrm{~dB}$ ) within the frequency bands where XPD $>25 \mathrm{~dB}$. In fact, the main bandwidth limitation of the antenna stems from the cross-polarization, not from the gain.

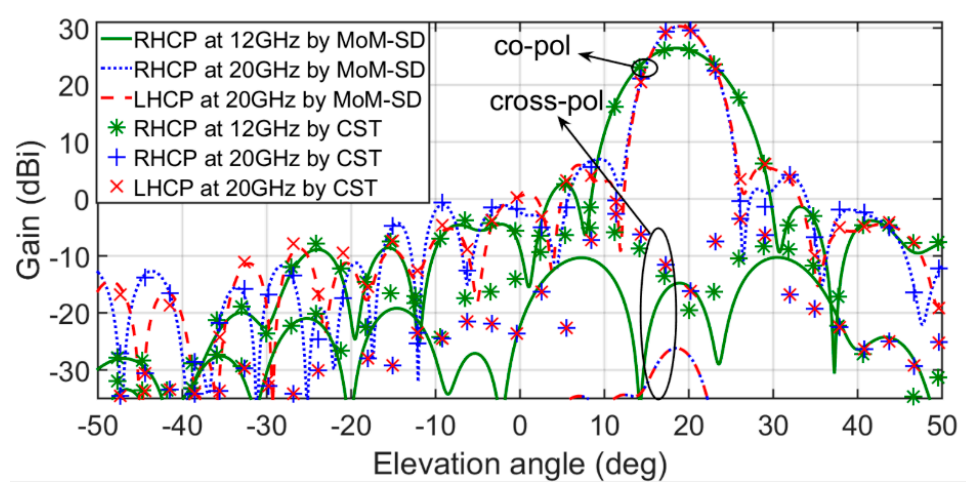

(a)

Figure 8. Cont. 




(b)

Figure 8. Main cuts of the simulated radiation patterns at $12 \mathrm{GHz}$ for right-hand circular polarization (RHCP) and $20 \mathrm{GHz}$ for both RHCP and left-hand circular polarization (LHCP). MoM-SD and CST results are shown. (a) Elevation cuts. (b) Azimuth cuts.

Table 1. Overall numerical performance of the designed circular polarization reflectarray that generates RHCP at $12 \mathrm{GHz}$, and RHCP and LHCP at $20 \mathrm{GHz}$.

\begin{tabular}{cccccc}
\hline $\begin{array}{c}\text { Frequency Band and } \\
\text { Polarization }\end{array}$ & Maximum Gain & $\begin{array}{c}\text { Bandwidth for XPD }> \\
\mathbf{2 5 ~ d B}\end{array}$ & $\begin{array}{c}\text { Antenna } \\
\text { Efficiency }\end{array}$ & $\begin{array}{c}\text { Maximum Side Lobe } \\
\text { Level }\end{array}$ & $\begin{array}{c}\text { Cross-Polar Level at } \\
\text { Central Frequency }\end{array}$ \\
\hline RHCP at $12 \mathrm{GHz}$ & $26.5 \mathrm{dBi}$ & $6.1 \%$ & $70 \%$ & $23.8 \mathrm{~dB}$ & $-11.2 \mathrm{dBi}$ \\
RHCP at $20 \mathrm{GHz}$ & $30.3 \mathrm{dBi}$ & $6 \%$ & $62 \%$ & $23.3 \mathrm{~dB}$ & $-26.3 \mathrm{dBi}$ \\
LHCP at $20 \mathrm{GHz}$ & $30.34 \mathrm{dBi}$ & $6 \%$ & $62 \%$ & $24.0 \mathrm{~dB}$ & $-26.3 \mathrm{dBi}$ \\
\hline
\end{tabular}

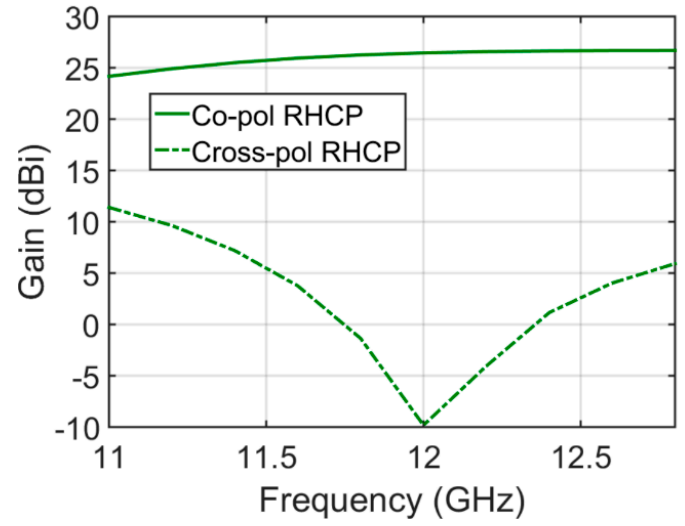

(a)



(b)

Figure 9. (a) Maximum of simulated gain versus frequency for frequencies closer $12 \mathrm{GHz}$. (b) Maximum gains versus frequency for frequencies closer $20 \mathrm{GHz}$.

The procedure used to design a reflectarray that radiates three circular polarization focused beams (one RCHP beam at $12 \mathrm{GHz}$, and two RHCP and LHCP beams at $20 \mathrm{GHz}$ ) in the same direction is extended to design a second reflectarray that radiates one RCHP beam at $12 \mathrm{GHz}$ in one direction, and two RHCP and LHCP beams at $20 \mathrm{GHz}$ in a different direction. In this latter case, we have used a circular reflectarray consisting of 716 elements arranged in a $30 \times 30$ square grid with a cell size of $8 \times 8 \mathrm{~mm}$, which is illuminated by two circular polarized feed-horns. One feed horn works at $12 \mathrm{GHz}$ for RHCP with its phase-center located at $\left(x_{f}, y_{f}, z_{f}\right)=(-0.0113,0.0,0.223)(\mathrm{m})$, while the other feed horn works at $20 \mathrm{GHz}$ for RHCP and LHCP with its phase-center located at $\left(x_{f}, y_{f}, z_{f}\right)=(-0.0616,0.0,0.247)(\mathrm{m})$. A similar configuration is used in [7]. Again, the radiation patterns of the feed horns are modeled as $\cos ^{10}(\theta)$ at $12 \mathrm{GHz}$ and $\cos ^{13}(\theta)$ at $20 \mathrm{GHz}$ [7]. In this case, the required phase shifts are computed in 
each element to produce a focused beam in the direction $\left(\phi_{\text {beam }}=0^{\circ}, \theta_{\text {beam }}=27^{\circ}\right)$ for $\mathrm{RHCP}$ at $12 \mathrm{GHz}$ and two focused beams in the direction $\left(\phi_{\text {beam }}=0^{\circ}, \theta_{\text {beam }}=14^{\circ}\right)$ for RHCP and LHCP at $20 \mathrm{GHz}$.

The results for the elevation cuts of the radiation patterns are shown in Figure 10. The reflectarray exhibits maximum gains of 27.62 and $32.29 \mathrm{dBi}$ at 12 and $20 \mathrm{GHz}$ respectively (with antenna efficiencies of $64 \%$ at $12 \mathrm{GHz}$ and $67 \%$ at $20 \mathrm{GHz}$ ). The cross-polar levels are roughly $-10 \mathrm{dBi}$ at $12 \mathrm{GHz}$ and $-26.1 \mathrm{dBi}$ at $20 \mathrm{GHz}$.

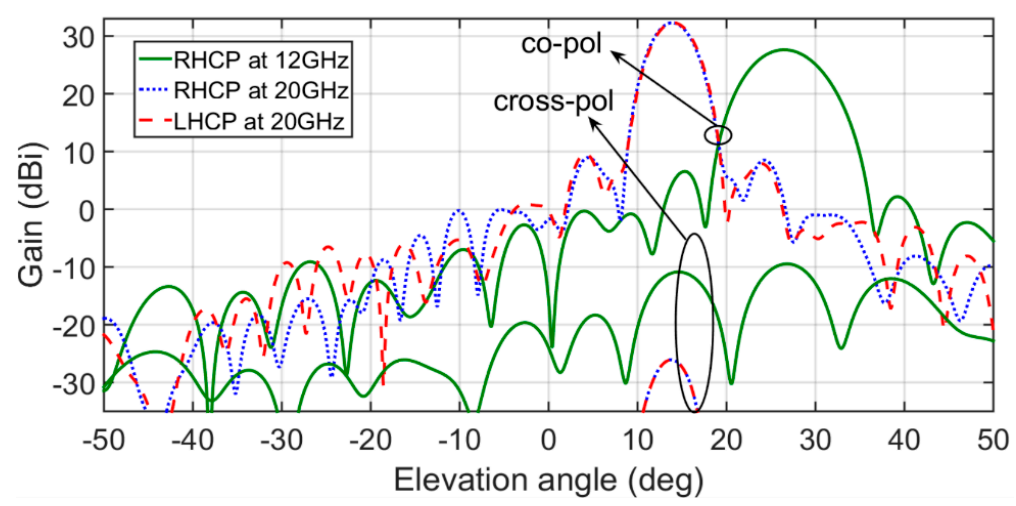

Figure 10. Simulated radiation patterns in elevation plane at $12 \mathrm{GHz}$ for $\mathrm{RHCP}$ and $20 \mathrm{GHz}$ for both RHCP and LHCP provided by designed reflectarray that radiates three circular polarization focused beams.

We have analyzed this last design reflectarray by taking into account tolerance errors of $5 \%$ variation of permittivity (i.e., $\varepsilon_{r 1}=\varepsilon_{r 2}=2.31$ ) and $+/-0.05 \mathrm{~mm}$ variations of the lengths of dipoles and arcs. These tolerance errors are higher than typical for dielectric layers material [20] and tolerance errors of mechanical PCB milling [31] in order to include the worst-case manufacturing scenario. These variations of length have been randomly added in each element of reflectarray. In Figure 11, a comparison of the resultant radiation patterns taking into account the tolerance errors and radiation patterns obtained in the elevation plane of Figure 10 is shown:

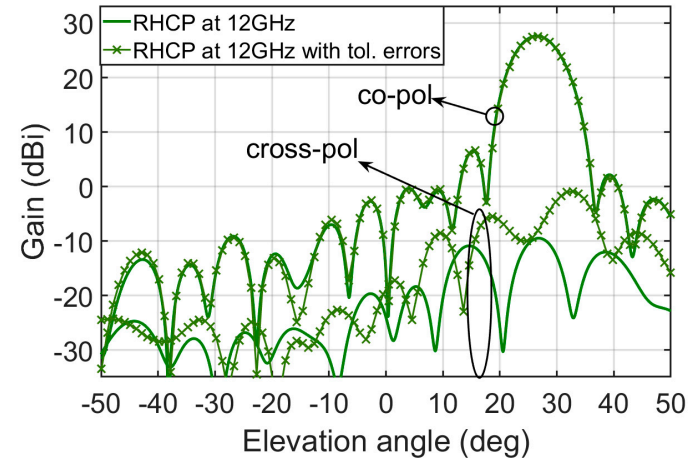

(a)

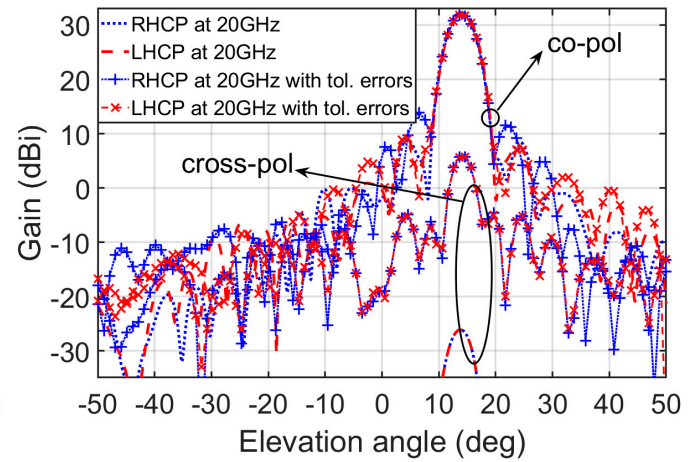

(b)

Figure 11. Comparison between the simulated radiation patterns in the elevation plane shown in Figure 10 and the simulated radiation patterns that take into account the tolerance errors of permittivity and length variations of the dipoles and arcs. (a) At $12 \mathrm{GHz}$ for RHCP. (b) At $20 \mathrm{GHz}$ for both RHCP and LHCP.

According with the results shown in Figure 11a, the tolerance errors produce insignificant variation of the copolar radiation pattern at $12 \mathrm{GHz}$ but a significant increment of cross-polar level is produced (from $-10 \mathrm{dBi}$ to $-0.9 \mathrm{dBi}$ of cross-polar gain). However, in Figure $11 \mathrm{~b}$ the side lobe levels of copolar radiation patterns change at $20 \mathrm{GHz}$ from $23.5 \mathrm{~dB}$ to $18 \mathrm{~dB}$ for $\mathrm{RHCP}$ and insignificant variation is 
produced for LHCP. The most significant variation is produced for cross-polar radiation patterns at $20 \mathrm{GHz}$ in both circular polarization when the cross-polar levels reach $5.7 \mathrm{dBi}$ of cross-polar gain due to tolerance errors. In order to avoid these significant variations of cross-polar level we recommend that the permittivity of dielectric layers was measured previous design reflectarray design and manufacturing the designed reflectarray with high accuracy machines, such as protolaser machines [31]. Furthermore, in $[17,18]$ measurements using similar reflectarray elements are provided (from 15 at $22 \mathrm{GHz}$ in [17] and from 19.2 at $29.75 \mathrm{GHz}$ in [18]) with acceptable results.

\section{Conclusions}

A two-layered reflectarray element is proposed consisting of a split ring surrounding two orthogonal sets of three parallel dipoles. This element makes it possible to design dual-band circular polarization reflectarrays radiating one circular polarization beam at Ku-band by means of VRT and two orthogonal circular polarization beams at Ka-band by ESVT for a satellite downlink. Rotations of split rings and different sizes of dipoles have been exploited as degrees of freedom by VRT and ESVT for designing focused beams dual band dual circular polarized reflectarrays. Until this work, these capabilities of the reflectarray element have been never exploited to focus the reflected beams in both circular polarizations for dual band applications. The split rings have been used to achieve single focused beam for circular polarization by VRT at $12 \mathrm{GHz}$, and the set of orthogonal dipoles have been used to achieve two focused beams for dual circular polarization by ESVT at $20 \mathrm{GHz}$.

The designed reflectarrays have been carried out under local periodicity assumption by means of in-house electromagnetic software that applies the Method of Moments in the Spectral Domain. Validations of the in-house electromagnetic software show significant CPU time savings with respect to CPU time consumption provided by commercial software of general purpose as CST electromagnetics software.

Parametric studies have shown when the dimensions of the split ring are adjusted by VRT at $12 \mathrm{GHz}$ and then fix the dipoles lengths by ESVT at $20 \mathrm{GHz}$, the resultant cross-polar levels are lower than when first fixing the dipoles lengths at $20 \mathrm{GHz}$, and then adjusting the split rings at $12 \mathrm{GHz}$. In this way, the design procedure is based on two stages: First VRT is applied based on rotations of the split ring with arc lengths adjustment. Second, the design procedure at Ka-band uses the ESVT, and is based on adjustment of the dipole's lengths for fixed values of the arc lengths/rotations obtained in the previous stage. Two different reflectarrays have been designed radiating RHCP at $12 \mathrm{GHz}$, and both RHCP and LHCP at $20 \mathrm{GHz}$, one of them with the same pointing direction at the two frequencies, and the other one with two different pointing directions. The simulated radiation patterns produced by reflectarrays show an efficiency around $65 \%$, and a bandwidth of $6 \%$ for a main beam cross-polarization discrimination of $25 \mathrm{~dB}$. The numerical results have been satisfactorily validated by CST software and tolerance errors study has been numerically carried out with acceptable results.

Author Contributions: Conceptualization, R.F., J.A.E.; methodology, R.F.; software, R.F., D.M.-d.-R., E.M.-d.-R. and R.R.B.; validation, R.F.; formal analysis, R.F. and R.R.B.; writing-original draft preparation, R.F.; writing-review and editing, R.F., J.A.E. and R.R.B.; visualization, R.F., D.M.-d.-R., E.M.-d.-R., J.A.E., R.R.B. and V.L.; supervision, J.A.E. and R.R.B.; project administration, J.A.E.; funding acquisition, J.A.E. All authors have read and agreed to the published version of the manuscript.

Funding: This work has been supported by the Spanish Ministry of Economy and Competitiveness under Project CICYT TEC2016-75103-C2-1-R, by the European Regional Development Fund (ERDF), by European Space Agency under ESTEC Contract No. 4000117113/16/NL/AF, and by “Junta de Andalucía” under Project P12-TIC-1435.

Conflicts of Interest: The authors declare no conflict of interest.

\section{References}

1. Yun, S.H.; Yom, I.B. Four band feed system with electrical traking capability for $\mathrm{Ku} / \mathrm{Ka}$ band bi directional antenna. In Proceedings of the 2008 IEEE Antennas and Propagation Society International Symposium, San Diego, CA, USA, 5-11 July 2008; pp. 1-4. 
2. Chang, Y.-C.; Hanlin, J. Commercial Ka and Ku bands reflector antennas. In Proceedings of the 2007 IEEE Antennas and Propagation Society International Symposium 2007, Honolulu, HI, USA, 9-15 June 2007; pp. 5175-5178. [CrossRef]

3. Bhutani, A.; Schaefer, J.; Pauli, M.; Scherr, S.; Goettel, B.; Nierlich, M.; Zwick, T. 3D metal printed Ku/Ka Band modified turn-stile junction orthomode transducer. In Proceedings of the 2016 Asia-Pacific Microwave Conference, New Delhi, India, 5-9 December 2016; pp. 1-4.

4. Huang, J.; Encinar, J.A. Reflectarray Antennas; Wiley: Piscataway, NJ, USA, 2008.

5. Martinez-De-Rioja, E.; Encinar, J.A.; Florencio, R.; Boix, R.R. Dual polarized reflectarray antenna to generate independent beams in $\mathrm{Ku}$ and Ka bands. In Proceedings of the 2016 10th European Conference on Antennas and Propagation (EuCAP), Davos, Switzerland, 10-15 April 2016; pp. 1-5.

6. Martinez-De-Rioja, E.; Encinar, J.A.; Florencio, R.; Boix, R.R. Reflectarray in K and Ka bands with independent beams in each polarization. In Proceedings of the 2016 IEEE International Symposium on Antennas and Propagation (APSURSI), Fajardo, Puerto Rico, 26 June-1 July 2016. [CrossRef]

7. Martinez-De-Rioja, E.; Encinar, J.; Barba, M.; Florencio, R.; Boix, R.R.; Losada-Torres, V. Dual Polarized Reflectarray Transmit Antenna for Operation in $\mathrm{Ku}$ - and Ka-Bands With Independent Feeds. IEEE Trans. Antennas Propag. 2017, 65, 3241-3246. [CrossRef]

8. Deng, R.; Xu, S.; Yang, F.; Li, M. An FSS-Backed Ku/Ka Quad-Band Reflectarray Antenna for Satellite Communications. IEEE Trans. Antennas Propag. 2018, 66, 4353-4358. [CrossRef]

9. Huang, J.; Pogorzelski, R. A Ka-band microstrip reflectarray with elements having variable rotation angles. IEEE Trans. Antennas Propag. 1998, 46, 650-656. [CrossRef]

10. Zhou, M.; Sørensen, S.B. Multi-spot beam reflectarrays for satellite telecommunication applications in Ka-band. In Proceedings of the 2016 10th European Conference on Antennas and Propagation (EuCAP), Davos, Switzerland, 10-15 April 2016; pp. 1-5.

11. Smith, T.; Gothelf, U.V.; Kim, O.S.; Breinbjerg, O. Design, manufacturing, and testing of a 20/30 GHz dual-band circularly polarized reflectarray antenna in submission. IEEE Antennas Wireless Propag. Lett. 2013, 12, 1480-1483. [CrossRef]

12. Deng, R.; Mao, Y.; Xu, S.; Yang, F. A Single-Layer Dual-Band Circularly Polarized Reflectarray With High Aperture Efficiency. IEEE Trans. Antennas Propag. 2015, 63, 1. [CrossRef]

13. Smith, T.; Gothelf, U.V.; Kim, O.S.; Breinbjerg, O. An FSS-Backed 20/30 GHz circularly polarized reflectarray for a shared aperture L- and Ka-band satellite communication antenna. IEEE Trans. Antennas Propagat. 2014, 62, 661-668. [CrossRef]

14. Pozar, D.M.; Metzler, T.A. Analysis of a reflectarray antenna using microstrip patches of different size. Electron. Lett. 1993, 29, 657-658. [CrossRef]

15. Encinar, J. Design of two-layer printed reflectarrays using patches of variable size. IEEE Trans. Antennas Propag. 2001, 49, 1403-1410. [CrossRef]

16. Florencio, R.; Encinar, J.A.; Boix, R.R.; Barba, M.; Toso, G. Flat Reflectarray That Generates Adjacent Beams by Discriminating in Dual Circular Polarization. IEEE Trans. Antennas Propag. 2019, 67, 3733-3742. [CrossRef]

17. Martinez-de-Rioja, D.; Florencio, R.; Encinar, J.A.; Carrasco, E.; Boix, R.R. Dual frequency reflectarray cell to provide opposite phase-shift in dual circular polarization with application multibeam satellite antennas. IEEE Antennas Wireless Propag. Lett. 2019, 18, 1591-1595. [CrossRef]

18. Martinez-de-Rioja, D.; Florencio, R.; Martinez-de-Rioja, E.; Arrebola, M.; Encinar, J.A.; Boix, R.R. Dual band reflectarray to generate to spaced beams in orthogonal circular polarization by variable rotation technique. IEEE Trans. Antennas Propag. 2020, 1-10, early access article. [CrossRef]

19. Huo, Y.; Dong, X.; Xu, W.; Yuen, M. Enabling Multi-Functional 5G and Beyond User Equipment: A Survey and Tutorial. IEEE Access 2019, 7, 116975-117008. [CrossRef]

20. Rogers Corporation. Available online: https://rogerscorp.com (accessed on 8 June 2020).

21. Florencio, R.; Boix, R.R.; Carrasco, E.; Encinar, J.A.; Losada, V.; Losada-Torres, V. Efficient numerical tool for the analysis and design of reflectarrays based on cells with three parallel dipoles. Microw. Opt. Technol. Lett. 2013, 55, 1212-1216. [CrossRef]

22. Florencio, R.; Boix, R.R.; Encinar, J.A. Efficient Spectral Domain MoM for the Design of Circularly Polarized Reflectarray Antennas Made of Split Rings. IEEE Trans. Antennas Propag. 2018, 67, 1760-1771. [CrossRef]

23. Mittra, R.; Chan, C.H.; Cwik, T. Techniques for analyzing frequency selective surfaces-a review. Proc. IEEE 1988, 76, 1593-1615. [CrossRef] 
24. Mesa, F.; Marqués, R.; Horno, M. A general algorithm for computing the bidimensional spectral Green's dyads in multilayered complex bianisotropic media: The equivalent boundary method. IEEE Trans. Microwave Theory Tech. 1991, 39, 1640-1649. [CrossRef]

25. Rahmat-Samii, Y. Useful coordinate transformations for antenna applications. IRE Trans. Antennas Propag. 1979, 27, 571-574. [CrossRef]

26. Florencio, R.; Boix, R.R.; Carrasco, E.; Encinar, J.A.; Barba, M.; Pérez-Palomino, G. Broadband reflectarrays made of cells with three coplanar parallel dipoles. Microw. Opt. Technol. Lett. 2014, 56, 748-753. [CrossRef]

27. Florencio, R.; Encinar, J.; Boix, R.R.; Perez-Palomino, G. Dual-polarisation reflectarray made of cells with two orthogonal sets of parallel dipoles for bandwidth and cross-polarisation improvement. IET Microw. Antennas Propag. 2014, 8, 1389-1397. [CrossRef]

28. CST Microwave Studio. Available online: www.cst.com (accessed on 8 June 2020).

29. Thomas, F.E.; John, L.V. Antenna Engineering Handbook; McGraw-Hill: Piscataway, NY, USA, 2007.

30. Zhou, M.; Sorensen, S.B.; Jorgensen, E.; Meincke, P.; Kim, O.S.; Breinbjerg, O. An Accurate Technique for Calculation of Radiation From Printed Reflectarrays. IEEE Antennas Wirel. Propag. Lett. 2011, 10, 1081-1084. [CrossRef]

31. LPKF Laser and Electronics. Available online: https://www.lpkf.com (accessed on 8 June 2020).

(C) 2020 by the authors. Licensee MDPI, Basel, Switzerland. This article is an open access article distributed under the terms and conditions of the Creative Commons Attribution (CC BY) license (http://creativecommons.org/licenses/by/4.0/). 